NORDITA 2000/11 HE

LPT-ORSAY 00-24

UNIL-IPT/00-03

February 2000

\title{
Fermion propagator in a nontrivial background field
}

\author{
Michael Joyce \\ LPT, Université Paris-XI, Bâtiment 211, F-91405 Orsay Cedex, France \\ E-mail: Michael.Joyce@th.u-psud.fr \\ Kimmo Kainulainen \\ NORDITA, Blegdamsvej 17, DK-2100, Copenhagen Ø, Denmark \\ E-mail: kainulai@nordita.dk \\ Tomislav Prokopec \\ Université de Lausanne, Institut de Physique Théorique, BSP, CH-1015 Lausanne, Suisse \\ E-mail: Tomislav.Prokopec@ipt.unil.ch
}

\begin{abstract}
We study the fermion propagator in a spatially varying classical background field, and show that, contrary to common wisdom, it may get nontrivial gradient corrections already at the first order in derivative expansion. This occurs whenever the fermion self-energy acquires a spatially (or temporally) varying pseudoscalar term, a simple example of which is given by a complex mass term $\hat{m}(x) \equiv m_{R}+i \gamma_{5} m_{I}$. Such effective mass terms arise for example in extensions of the Standard Model during the electroweak transition, and they are crucial in providing the CP-violation necessary for electroweak baryogenesis.
\end{abstract}




\section{Introduction}

Often in applications of quantum field theories, it is desirable to treat a part of the problem semiclassically and compute the quantum corrections in a controlled expansion around the classical solutions. This applies to a broad spectrum of problems, including for example the Fermi liquid theory, as well as the study of the expanding and cooling plasma in the heavy ion collisions [1, 2]. In these, and in many other cases, it is often sufficient to assume that the background is very slowly varying and to treat the problem at leading order in gradients. Such an adiabatic (quasiparticle) approximation may make sense if the physical effect of the background one is interested in is already present at the trivial order. However, there are situations where a semiclassical approximation is justified, but the system displays an important physical effect only at a nontrivial order in gradients. An important example of this occurs at the electroweak transition, where the problem is to study particle propagation in the presence of a spatially varying, CP-violating Higgs condensate. The CP-violating effects of the condensate on the plasma dynamics arise only at the first nontrivial order in gradient expansion [3, 4, 5, 6]. The induced perturbations in the particle and antiparticle densities, coupled with the weak anomaly, lead to a local baryon production mechanism, capable of creating the observed matter-antimatter asymmetry of the Universe [0, 6].

Unfortunately, no consistent derivation of the semiclassical quantum Boltzman equation for a fermionic field beyond the trivial order in gradients exist in the literature. Moreover, there exists a well known no-go theorem [1], according to which first nontrivial corrections to fermion propagator arise only at second order in derivative expansion. This is in apparent contrast with the classical force mechanism (CFM) for baryogenesis [4, 6], according to which the nontrivial effects on fermion propagation are felt already at the first order in gradients. In this letter we show that the no-go theorem does not hold universally, but instead, whenever the fermion self energy contains a pseudoscalar term, there are corrections to the propagator already at the first order in gradients. A simple example of such a term is provided by a Dirac field with a complex spatially (or temporally) varying mass term of the form

$$
\hat{m}(x)=m_{R}(x)+i \gamma_{5} m_{I}(x) \equiv|m| e^{i \gamma^{5} \theta}
$$

This term could be of course replaced by generic scalar and pseudoscalar operators; we retain 
the present notation however, because of its clear physical implications. Namely, this type of term has precisely the form relevant for baryogenesis, where such an effective mass term is induced by a coupling of the fermion with a spatially varying complex scalar field condensate during a first order phase transition. Here we shall consider a simple toy model with the lagrangian

$$
\mathcal{L}=i \bar{\psi} \not \partial \psi-\bar{\psi}_{L} m \psi_{R}-\bar{\psi}_{R} m^{*} \psi_{L}+\mathcal{L}_{\text {int }},
$$

where $m=m_{R}+i m_{I}$ is a complex mass. The detailed form of the interaction lagrangian $\mathcal{L}_{\text {int }}$ is not relevant for us here. Realistic cases studied in literature in connection with the CFM baryogenesis include the two doublet extension of the standard model [ [| and the chargino sector of the minimal supersymmetric standard model [6].

This letter is a part of a series [8, 9, 10] dedicated to derivation of semiclassical transport equations for quantum fields in varying backgrounds beyond the trivial order in gradients. We first explicitly construct the fermion propagator for the theory (2) to the first nontrivial order in gradients. Corrections appear as higher order poles multiplied by derivatives of the complex phase of the mass. The position of the poles is not shifted however, and the physical content of the correction terms only becomes apparent after consideration of spectral integrals over test functions by a technique developed in [8]. It will be shown that the spectral function, while not expressible as a sum of ordinary delta-functions even in the on-shell limit, projects a test function to a sharp, but shifted energy shell. We then show that this

shell corresponds to the physical semiclassical dispersion relation of a standard WKB wave function, and describe how the current computed using the spectral function representation can be related to motion of WKB wave packets.

\section{Propagator}

Our first task is to solve the retarded and advanced propagators for the fermionic field $\psi$ in the weak coupling approximation and in an expansion in gradients of the slowly varying mass terms. We will work in the Keldysh closed time contour (CTC) formalism [11, 12, 1], 
and the basic quantity of interest is the path-ordered propagator

$$
i G_{\mathcal{C}}^{\psi}(x, y)=\left\langle T_{\mathcal{C}}[\psi(y) \bar{\psi}(x)]\right\rangle .
$$

Writing the Schwinger-Dyson equations for $G_{\mathcal{C}}$ in the real time formulation implies the following formally exact equations for the retarded and advanced propagators $G^{r, a}$ in the Wigner representation [1]

$$
e^{-i \diamond}\left\{\left(i G_{0}\right)^{-1}\right\}\left\{i G^{r, a}(k ; X)\right\}=1,
$$

where $\diamond\{f\}\{g\} \equiv \frac{1}{2}\left(\partial_{X} f \cdot \partial_{k} g-\partial_{k} f \partial_{X} g\right)$ and the tree level propagator is given by

$$
\left(i G_{0}^{r, a}\right)^{-1} \equiv \not k-m_{R}(X)-i \gamma_{5} m_{I}(X)-\Sigma^{r, a} \pm i s_{\omega} \epsilon,
$$

where $s_{\omega}=\operatorname{sign}(\omega), k$ denotes the canonical 4-momentum and $X \equiv(x+y) / 2$ the average position. From now on we shall assume a weak coupling limit and neglect the self-energy $\Sigma^{r, a}=\Sigma_{R} \mp i \omega \Gamma$; in particular we thus consider the on-shell limit $\Gamma \rightarrow 0$. We will also suppress the $i \epsilon$-prescription which differentiates between $G^{r, a}$ even when $\Gamma \rightarrow 0$, which is introduced as a mnemonic tool to maintain information about the integration path required to fully specify how $G$ acts operationally. A formal solution to equation (5) can easily be found in the gradient approximation. Indeed, to first order in gradients we have:

$$
i G=i G_{0}-i G_{0} i \diamond\left\{\left(i G_{0}\right)^{-1}\right\} i G_{0}\left\{\left(i G_{0}\right)^{-1}\right\} i G_{0},
$$

where the free propagator is given by

$$
i G_{0}=\frac{1}{k^{2}-|m|^{2}}\left(\not k+m_{R}(X)-i \gamma_{5} m_{I}(X)\right) .
$$

After some algebra one can write the expression (6) into the following covariant form:

$$
i G=i G_{0}+\frac{1}{\left(k^{2}-|m|^{2}\right)^{2}}\left[i\left(k \cdot \partial_{X}-\not \not_{X}\right)|m| e^{-i \theta \gamma_{5}}+\gamma^{5}|m|^{2} \not_{X} \theta\right] .
$$

There is clearly a nonvanishing correction to the propagator at the leading nontrivial order. This is not in disagreement with the no-go theorem by [1], because, as we shall see, all nontrivial consequences of (8) are proportional to the complex pseudoscalar mass (or more 
generally to a pseudoscalar self-energy function), which was not considered in Ref. [1]. An important point to observe about (8) is that the pole of the propagator is not shifted, but instead a new second order pole, proportional to gradients of $m$ has appeared. This behaviour is analogous to one previously observed in the case of a scalar field [8].

For simplicity we shall from now on restrict ourselves to the case of a planar symmetry with only spatially varying mass: $m=m\left(x_{3}\right)$. In this case one can Lorentz boost to the frame in which $\mathbf{k}_{\|}=\mathbf{0}$, so that $\omega \rightarrow \tilde{\omega} \equiv s_{\omega}\left(\omega^{2}-\mathbf{k}_{\|}^{2}\right)^{1 / 2}$. With these definitions Eq. (8) simplifies to

$$
\begin{aligned}
i \gamma^{0} G & =\frac{1}{z}\left(\tilde{\omega}-k_{3} \gamma^{5} S^{3}+\gamma^{0} m_{R}-i \gamma^{0} \gamma^{5} m_{I}\right) \\
& -\frac{1}{z^{2}}\left[i \tilde{\omega}\left(m_{R}^{\prime} \gamma^{0} \gamma^{5}-i m_{I}^{\prime} \gamma^{0}\right)+|m|^{2} \theta^{\prime}\right] S^{3},
\end{aligned}
$$

where we combined various $\gamma$-matrices into the spin operator $S^{3}=\gamma^{0} \gamma^{3} \gamma^{5}$, which measures the spin in the $\hat{\mathbf{k}}_{3}$-direction and in the frame in which $\mathbf{k}_{\|}=\mathbf{0}$, and we used the definition $z \equiv$ $k^{2}-|m|^{2}$. Allowing $z$ a small complex value, one sees that (9) yields $G^{r}\left(G^{a}\right)$ for $\operatorname{Im}(z)<0(>$ 0). A further very important simplification arises from the fact that pseudoscalar interactions conserve spin (one can show that the spin-operator $S^{3}$ commutes with the propagator $G$ ). The problem then becomes diagonal in spin and, by replacement $S^{3} \rightarrow s$, the $4 \times 4$ structure of (9) becomes block-diagonal, so that the problem can be reduced to a $2 \times 2$ problem. In the $2 \times 2$ formalism, it is more convenient to use the Pauli matrices for the spinor algebra, which can be effected by the following identifications:

$$
\gamma^{0} \rightarrow \sigma^{1}, \quad-i \gamma^{0} \gamma^{5} \rightarrow \sigma^{2} \quad-\gamma^{5} \rightarrow \sigma^{3}
$$

where we have taken the chiral representation for the $\gamma$ matrices. In this way (9) can be recast in a particularly simple form:

$$
\begin{aligned}
i \sigma^{1} G_{s} & =\frac{1}{z}\left(\tilde{\omega}+s k_{3} \sigma^{3}+m_{R} \sigma^{1}+m_{I} \sigma^{2}\right) \\
& -\frac{s}{z^{2}}\left[\tilde{\omega}\left(m_{I}^{\prime} \sigma^{1}-m_{R}^{\prime} \sigma^{2}\right)+|m|^{2} \theta^{\prime}\right] \\
& \equiv b_{0}+\mathbf{b} \cdot \sigma .
\end{aligned}
$$

From the last expression in particular it is evident that there exists a basis in which the propagator $G$ is diagonal $G_{s} \rightarrow G_{d, s}=b_{0}+s \operatorname{sign}(\tilde{\omega})|\mathbf{b}| \sigma^{3}$. In the Dirac notation the propagator 
then reads

$$
i \gamma^{0} G_{d}=s_{\tilde{\omega}}\left(\frac{|\tilde{\omega}|}{z}-\frac{s s_{\tilde{\omega}}|m|^{2} \theta^{\prime}}{z^{2}}\right)\left(1-s_{\tilde{\omega}} S^{3} \gamma^{5}\right),
$$

where $s_{\tilde{\omega}} \equiv \operatorname{sign}(\tilde{\omega})$. Physically the equation (12) means that the states with equal $s s_{\tilde{\omega}}$ propagate identically. Formally, this is expressed by the projector $\left(1-s_{\tilde{\omega}} S^{3} \gamma^{5}\right) / 2$, which identifies the states with equal entries on the diagonal. In particular this means that particles and antiparticles with opposite spin have identical spectral functions. However, for particles and antiparticles of same spin (helicity) the spectral functions differ by an amount proportional to the CP-violating angle $\theta^{\prime}$. This is how in the context of baryogenesis CP-violating backgrounds induce bias in the particle and antiparticle distributions. In order to understand how this bias propagates in a plasma, it is then necessary to study the relevant quantum trasport equations with the effect of collisions included [13].

\section{Spectral integrals}

To study the physical consequences of the propagator (8), we now consider spectral integrals over test functions [8], representing some generic observables, such as the generalized particle distribution function. We define:

$$
G[\mathcal{T}] \equiv \operatorname{Tr} \frac{i}{2 \pi} \int_{0}^{\infty} d \omega \gamma^{0} \mathcal{A} \mathcal{T}
$$

where $\mathcal{A} \equiv \frac{i}{2}\left(G^{r}-G^{a}\right)$ is the spectral function, $\mathcal{T}$ is a test function, and the trace is taken over the Dirac indices. Because scalar and pseudoscalar backgrounds considered here conserve spin, we can take $\mathcal{T}$ to be diagonal in the spin space and a scalar function in a spin $2 \times 2$ block. For backgrounds that violate spin this analysis would have to be generalized to include spin mixing. The $\gamma^{0}$-factor in Eq. (13) is added to make the projection operator explicitly hermitean. The spectral function $\mathcal{A}$ carries information on the physical spectrum of excitations in the system. In particular to zeroth order in gradients it becomes a simple on-shell projector Đ ( $c f$. Eq. (7)) :

$$
\mathcal{A} \rightarrow \frac{\not k+m_{R}-i \gamma_{5} m_{I}}{2 \omega_{0}}\left[\delta\left(\omega-\omega_{0}\right)-\delta\left(\omega+\omega_{0}\right)\right], .
$$

\footnotetext{
${ }^{1}$ Note that we are assuming the on-shell limit and neglecting possible thermal and/or vacuum corrections, so that $\Sigma^{r, a}=0$.
} 
where $\omega_{0}=\left(k_{3}^{2}+|m|^{2}\right)^{1 / 2}$. In a spatially varying background the spectral function can no longer be expressed in terms of $\delta$-functions projecting onto the physical energy shells. As discussed in [8], it can however be written as a sum of projectors onto complex shells, but this is not the approach we will pursue here. Instead, by extending the momentum to the complex plane, the integral (13) can be turned into a contour integral over the variable $z \equiv k^{2}-|m|^{2}[8]:$

$$
G[\mathcal{T}]=\operatorname{Tr} \frac{i}{4 \pi} \int_{\mathcal{C}_{0}} d z \frac{i \gamma^{0} G \mathcal{T}}{\sqrt{\omega_{0}^{2}+z}},
$$

where $G$ is the propagator $(8)$, and $\mathcal{C}_{0}$ is a contour encircling the pole at $z=0\left(\omega=\omega_{0}\right)$ once clockwise. We can then compute (15) by making use of the residue theorem:

$$
G[\mathcal{T}]=\operatorname{Tr} \operatorname{Res}_{z=0}\left[\frac{i \gamma^{0} G \mathcal{T}}{2 \sqrt{\omega_{0}^{2}+z}}\right] .
$$

Because the highest pole in the expression (\$) is of the second order, only the terms up to the linear order in $z$ in the expansions of various terms appearing in (16) contribute. In particular the gradient expansion in $\partial_{k_{z}} \mathcal{T}$ then terminates at first order. That is to say that all $\partial_{k_{z}}^{l} \mathcal{T}$-terms for $l>1$ are of at least second order in gradients. An important consequence of this truncation is that a complete description of a fermionic plasma, consistent to first order in gradients, requires at most two independent distribution functions, in contrast to the four distribution functions required for a full description of the bosonic case at the lowest nontrivial order in gradients [10].

It is now a simple matter to compute the residue in Eq. (16) of the propagator (11) or (12). (For notational simplicity from now on we shall denote $\tilde{\omega}$ by $\omega$.) To the order we are working

$$
\begin{aligned}
G[\mathcal{T}] & =\mathcal{T}_{0}+\frac{s s_{\omega}|m|^{2} \theta^{\prime}}{2 \omega_{0}^{2}}\left(\mathcal{T}_{0}-\omega_{0} \mathcal{T}_{0}^{\prime}\right) \\
& =\frac{\omega_{0}}{\omega_{\mathrm{sc}}} \mathcal{T}_{0}\left(\omega_{\mathrm{sc}}\right),
\end{aligned}
$$

where $\mathcal{T}_{0} \equiv \mathcal{T}\left(\omega_{0}\right), \mathcal{T}_{0}^{\prime} \equiv\left(\partial_{\omega} \mathcal{T}\right)\left(\omega_{0}\right)$, and we have defined the semiclassical energy as

$$
\omega_{\text {sc }} \equiv \omega_{0}-\frac{s s_{\omega}|m|^{2} \theta^{\prime}}{2 \omega_{0}^{2}} .
$$

The test function thus still gets projected to a sharply defined energy shell, which is however shifted with respect to the lowest order shell $\omega_{0}$. 


\section{Current}

As a physically motivated application of the above, we now consider a current of spin states. In the present formalism this can be written as follows:

$$
j_{s}^{\mu}(X)=\operatorname{Tr} \int \frac{d^{4} k}{(2 \pi)^{4}} i \gamma^{\mu} G^{<}(k ; X) P_{s},
$$

where $P_{s}=\left(1+s S^{3}\right) / 2$ is the spin projector and $G^{<}(k ; X)$ is the quantum Wigner function. Assuming the decomposition $i G^{<}(k ; X) P_{s}=\mathcal{A} P_{s} n_{s}$, where $n_{s}$ are generalized particle distribution functions of spin states, and inserting this expression into (19) it is a simple matter to show that

$$
\begin{aligned}
& j_{s}^{0}(X)=2 \int \frac{d^{3} k}{(2 \pi)^{3}} \frac{\omega_{0}}{\omega_{\mathrm{sc}}} f_{s}^{\mathrm{sc}}(\mathbf{k} ; X) \\
& j_{s}^{3}(X)=2 \int \frac{d^{3} k}{(2 \pi)^{3}} \frac{k_{3}}{\omega_{0}} f_{s}^{0}(\mathbf{k} ; X),
\end{aligned}
$$

where the (momentum space) semiclassical distribution function $f_{s}^{\mathrm{sc}}(\mathbf{k} ; X) \equiv n_{s}\left(\omega_{\mathrm{sc}}, \mathbf{k} ; X\right)$ is the projection of $n_{s}$ onto the semiclassical shell $\omega=\omega_{\mathrm{sc}}$, while the distribution function $f_{s}^{0}(\mathbf{k} ; X) \equiv n_{s}\left(\omega_{0}, \mathbf{k} ; X\right)$ is the projection of $n_{s}$ onto the unshifted shell $\omega=\omega_{0}$. Further, in Eq. (20) we have reintroduced the dependencies on the trivial perpendicular directions $\mathbf{k}_{\|}$. Finally, changing the variables $k_{3} \rightarrow k_{\mathrm{sc}}, \omega_{\mathrm{sc}} \rightarrow \omega\left(d k_{3} \rightarrow d \omega\left(\partial \omega_{\mathrm{sc}} / d k_{3}\right)^{-1}\right)$ in the first integral, and $k_{3} \rightarrow k_{0} \equiv\left(\omega^{2}-|m|^{2}\right)^{1 / 2}, \omega_{0} \rightarrow \omega\left(d k_{3} \rightarrow d \omega \omega_{0} / k_{3}\right)$ in the second integral, the current reduces to

$$
j_{s}^{\mu}=2 \int \frac{d \omega d^{2} k_{\|}}{(2 \pi)^{3}}\left(\frac{\omega}{k_{\mathrm{sc}}} f_{s}^{\mathrm{sc}}, \hat{\mathbf{k}} f_{s}^{0}\right),
$$

where the distribution functions $f_{s}^{\mathrm{sc}}=f_{s}^{\mathrm{sc}}\left(\omega, \mathbf{k}_{\|} ; X\right) \equiv n_{s}\left(\omega, k_{\mathrm{sc}}, \mathbf{k}_{\|} ; X\right)$ and $f_{s}^{0}=f_{s}^{0}\left(\omega, \mathbf{k}_{\|} ; X\right)$ $\equiv n_{s}\left(\omega, k_{0}, \mathbf{k}_{\|} ; X\right)$ are now defined on the energy phase space $\left(\omega, \mathbf{k}_{\|} ; X\right)$. The semiclassical momentum $k_{\mathrm{sc}}=k_{\mathrm{sc}}\left(\omega, \mathbf{k}_{\|} ; X\right)$ in Eq. (21) is the one obtained by inverting the semiclassical energy-momentum relation (18):

$$
k_{\mathrm{sc}}=k_{0}\left(1+\frac{s s_{\omega}|m|^{2} \theta^{\prime}}{2 k_{0} \omega^{2}}\right) .
$$

Of course, in equilibrium the spatial currents cancel. However, if the preferred coordinate frame is boosted with respect to the plasma rest frame, as is the case for a propagating phase 
transition front, then there are net currents flowing through the space. One can then determine how these induced currents are affected by the presence of a background mass term (11). Since particles and antiparticles of a given spin (helicity) $s$ and energy $|\omega|$ have different effective momenta $k_{\mathrm{sc}}$, the corresponding particle and antiparticle densities $j_{s}^{0}$ are different in the region of a spatially varying background. This is how local particle-antiparticle asymmetric density and current profiles get created by a spatially varying and CP-violating background, which is the phenomenon at the heart of most electroweak baryogenesis mechanisms.

\section{$5 \quad$ Field theoretical vs WKB approach}

Let us now make a connection between the above results and the dispersion relation obtained by using the WKB method. Through a standard calculation [4, 6], one finds

$$
k_{\mathrm{wkb}}=k_{0}+\frac{s s_{\omega} \theta^{\prime}}{2 k_{0}}\left(\omega \pm s k_{0}\right)+\alpha^{\prime}, \quad k_{0}=\sqrt{\omega^{2}-|m|^{2}}
$$

where the arbitrary function $\alpha^{\prime}$ follows from the reparametrization invariance of the theory given by the lagrangian (2) invariant under (global) $U(1)$-transformations $\psi \rightarrow e^{i \alpha} \psi$. It is then obvious that $k_{\mathrm{wkb}}$ cannot represent a physical quantity. The group velocity

$$
v_{g} \equiv\left(\partial_{\omega} k_{\mathrm{wkb}}\right)^{-1}
$$

corresponding to the stationary phase of the WKB wave packet, is a well defined physical

quantity however. The physical momentum of a WKB state is then given by $k_{\text {phys }}=\omega v_{g}(\omega)$. Computing the derivative (24) one immediately finds the physical reparametrization invariant WKB dispersion relation

$$
k_{\mathrm{phys}} \equiv \omega v_{g}=k_{0}\left(1+\frac{s s_{\omega}|m|^{2} \theta^{\prime}}{2 k_{0} \omega^{2}}\right),
$$

which is identical to Eq. (22). With this the semiclassical momentum (22) can be reinterpreted as the physical momentum of a WKB state. In this sense we have established the equivalence between the physical WKB dispersion relation and the field theoretical energymomentum shell corresponding to the propagator (8), or equivalently (12).

We now return to discuss the current in Eq. (21). The difference between the WKB approach and the field theoretical one presented here is the fact that there are two mutually 
independent distribution functions $f_{s}^{\text {sc }}$ and $f_{s}^{0}$. In the same spirit as it was done for the scalar field in Ref. [10], we may introduce a coherent quantum density $f_{s}^{\mathrm{qc}}=f_{s}^{\mathrm{sc}}-f_{s}^{0}$, which is a dynamical measure of quantum coherence on phase space between the semiclassical and the unshifted shell. At the leading order in gradients $f_{s}^{\mathrm{qc}}$ of course vanishes. In analogy to the scalar field case we then expect that in the frequent scattering limit $f_{s}^{\text {qc }}$ gets suppressed and can be consistently neglected. Assuming this is the case, we may then set $f_{s}^{\text {qc }} \rightarrow f_{s}$, $f_{s}^{0} \rightarrow f_{s}$, so that finally the current (21) reduces to the following form

$$
j_{s}^{\mu} \rightarrow 2 \int \frac{d \omega d^{2} k_{\|}}{(2 \pi)^{3}}\left(\frac{1}{v_{g}} ; \hat{\mathbf{k}}\right) f_{s} .
$$

This limit is in perfect agreement with the naive WKB result [14], implying the following simple physical interpretation: when quasiparticles arrive into a region of a spatially varying background, they either speed up or slow down. If they slow down for example, the local particle density increasess proportionally to the factor $1 / v_{g}$. At the same time the flux, given by the spatial part of the current, remains unchanged, as it should. To prove (26) rigorously requires derivation and solution of the relevant quantum transport equations to determine selfconsistently the distribution functions $f_{s}^{\text {sc }}$ and $f_{s}^{0}$. This problem will be further considered in a forthcoming work [13].

While it was to be expected that the field theoretical calculation, unlike the WKBcomputation, is automatically invariant under the field redefinition $\psi \rightarrow e^{i \alpha} \psi$, the fact that the two methods lead to identical dispersion relations and currents (in the frequent scattering limit) is highly nontrivial. Indeed, it should be stressed that the physical WKBshell has emerged from the field theoretical calculation indirectly, not as a simple shift of the propagator poles, but rather by the operation of a modified spectral projector on test functions, which is effected via the technique of spectral integrals.

\section{Conclusions}

We have studied the fermion propagator coupled with a spatially varying classical background field. We represented this coupling by a spatially varying complex mass term, and proved that the propagator receives nontrivial corrections already at the first order in the gradients 
of the mass term $m$. For this result it was crucial that $m$ contains a nonvanishing complex pseudoscalar piece. We then explicitly constructed the fermion propagator and the associated spectral function to the first order in gradients. The gradient corrections were shown to arise, not as shifts in the poles, but rather as additional higher order poles multiplied by the gradients of the complex phase of $m$. We then considered a spectral integral of a simple test function, and showed that it nevertheless gets projected to a sharp energy shell, which acquires a spin dependent shift with respect to the lowest order shell. The new shell was then shown to coincide with the physical dispersion relation of a WKB-state. We also computed the current in the field theoretical way, and showed that it can be interpreted in terms of moving WKB wave packets. We note that it is straightforward to generalize our results to a generic pseudoscalar self-energy term and to temporally varying fields.

Here we have concentrated on the propagation of fermions in a nontrivial backgrounds. The next logical step is to study the transport properties in a plasma containing fermions. Drawing on the insights obtained from the study of the scalar case [10], the results presented here suggest that a consistent description of transport at nontrivial order in gradient expansion will require a set of coupled equations for number density and an additional function describing quantum coherence in phase space. We also expect that in the frequent scattering limit the quantum coherence effects are suppressed, and an effective quasiparticle picture is recovered [13].

\section{Acknowledgements}

We wish to thank Felipe Freire for collaboration at the early stages of this project, and Dietrich Bödecker for illuminating discussions. KK thanks CERN for hospitality during the completion of this work.

\section{References}

[1] P. Henning, Phys. Rep. 253235 (1995). 
[2] For a review see for example U. Heinz, Invited talk at 14th International Conference on Ultrarelativistic Nucleus-Nucleus Collisions (QM 99), Torino, Italy, 10-15 May 1999, nucl-th/9907060.

[3] N. Turok and J. Zadrozny, Nucl. Phys. B358 471 (1991); A.G. Cohen and D.B. Kaplan, Phys. Lett. B263 86 (1991).

[4] M. Joyce, T. Prokopec and N. Turok, Phys. Lett. B338 269 (1994); Phys. Rev. Lett. 751695 (1995), ERRATUM-ibid. 753375 (1995); Phys. Rev. D53 2930 (1996); Phys. Rev. D53 2958 (1996).

[5] P. Huet and A.E. Nelson, Phys. Rev. D53 4578 (1996); M. Carena, M. Quiros, A. Riotto, I. Vilja and C.E.M. Wagner, Nucl. Phys. B503 387 (1997).

[6] J. Cline, M. Joyce and K. Kainulainen, Phys. Lett. B417 79 (1998).

[7] For review see for example V. Rubakov and M.E. Shaposhnikov, Phys. Usp. 39, 461 (1996).

[8] M. Joyce, K. Kainulainen, and T. Prokopec, Phys. Lett. B468 128 (1999).

[9] M. Joyce, K. Kainulainen, and T. Prokopec, hep-ph/9906413, proceedings of SEWM-98, World Scientific 1999.

[10] M. Joyce, K. Kainulainen, and T. Prokopec, hep-ph/9910535, to appear in Phys. Lett. B.

[11] J. Schwinger, J. Math. Phys. 2407 (1961); L.V. Keldysh, Zh. Eksp. Teor. Fiz. 471515 (1964) [Sov. Phys. JETP 20235 (1965)].

[12] L.P. Kadanoff and G. Baym, Quantum Statistical Mechanics, Benjamin Press, New York (1962). For review see for example ref. [1], P. Danielewicz, Ann. Phys. 152239 (1984).

[13] M. Joyce, K. Kainulainen, and T. Prokopec, in progress.

[14] K. Kainulainen, to be published in the proceedings of Cosmo-99, 1999, Trieste, Italy. 